\title{
Evaluating the effects of Bt protein Vip3Aa on immature Propylea japonica (Thunberg) (Coleoptera: Coccinellidae)
}

Intazar Ali ${ }^{1,2^{*}}$, Shuai Zhang ${ }^{1,4}$, Asif Sajjad², Ghulam Khaliq ${ }^{3}$, Muhammad Basit ${ }^{5}$, Ghulam Ali Bugti ${ }^{3}$, Hafiz Tassawar Abbas ${ }^{3}$, Naeem Abbas ${ }^{6}$ and Jin-Jie Cui ${ }^{1}$

\begin{abstract}
Background: Genetically modified (GM) crops have become a common tool for managing insect pests of economic importance. However, before their commercialization, bio-safety evaluation on non-target arthropods such as pollinators and biological control agents is a pre-requisite to save services rendered by them in agro-ecosystem.

Results: In the present experiment, the potential adverse effects of Bt protein (Vip3Aa) were studied on the life table parameters (pupation and emergence rates, 7-day larval weights, larval development time, and adult weight) of a predatory beetle, Propylea japonica (Thunberg) (Coleoptera: Coccinellidae) in the laboratory. P. japonica was exposed to Bt protein incorporated in synthetic diet at the rate of $200 \mu \mathrm{g} / \mathrm{g}$ of fresh weight of diet. Temporal stability, bioactivity and the uptake of insecticidal protein were confirmed by enzyme-linked immunosorbent assay and a sensitive insect bioassay. The results indicated that pupation and adult emergence rates did not differ significantly from that of negative control (pure synthetic diet). Similarly, the fresh weight of male and female adults emerged in Vip3Aa treatment ( $2.87 \mathrm{mg}$ and $3.86 \mathrm{mg}$, respectively) did not vary significantly from that of the negative control treatment $(2.66 \mathrm{mg}, 3.75 \mathrm{mg}$ ). However, the time taken by the larvae to complete their development (12.70 days) was significantly extended than that of the negative control (10.21 days). In contrast, the tested life-table parameters of $P$. japonica were adversely affected when fed on synthetic diet containing boric acid (positive control) indicating experimental fitness to detect adverse effects.
\end{abstract}

Conclusions: On the basis of the results, it is suggested that GM crops expressing Bt protein (Vip3Aa) are safe for non-target insects such as P. japonica and are compatible with other biological programs for the integrated management of insect pests.

Keywords: Bio-safety evaluation, Dietary exposure test, Propylea japonica, Vip3Aa protein

\section{Background}

Extensive use of insecticides not only increases resistance in insect pests but also reduces the bio-diversity of non-target arthropods (NTA) (Jafar et al. 2013). To overcome these problems, scientists have modified the crops genetically using the genes of Bacillus thuringiensis

\footnotetext{
*Correspondence: intazar_1228@yahoo.com

${ }^{1}$ State Key Laboratory of Cotton Biology, Institute of Cotton Research,

Chinese Academy of Agricultural Sciences, Anyang 455000, China

Full list of author information is available at the end of the article
}

(Berliner) with the aim to provide enhanced resistance in plants against insect pests without affecting NTA. Bt synthesizes potent insecticidal proteins, such as the Cry proteins during sporulation, commonly used in GM crops (Palma et al. 2014). In addition, Bt secretes numerous vegetative insecticidal proteins (Vip) during its vegetative phase. These proteins have also shown a broad-spectrum activity against Lepidoptera without sharing target binding sites with Cry proteins in the target insect midgut. Therefore, these $B t$ proteins are considered a good complement to Cry proteins (Chakrabarty et al. 2020). The 
genes coding for expressing various Cry and Vip3 proteins have been incorporated genetically in GM plants to protect them from insect pests of economic importance (Kumar et al. 2020).

GM crops are now being opted as a significant tool to manage the insect pests of economic concerns worldwide with less number of broad-spectrum chemical applications (Klümper and Qaim 2014), and these GM crops are generally considered safe to NTA (Yin et al. 2020). However, there is a possibility that GM crops might have lethal or sub-lethal effects on the fitness of NTA found abundantly in cropping ecosystems, and playing a substantial role in the suppression of insect pests without or with little human assistance. Thus, it is considered imperative to evaluate GM crops for their potential adverse effects on NTA before their release for wide commercial cultivation in the field (Romeis et al. 2008).

The Japanese ladybird, Propylea japonica (Thunberg) (Coleoptera: Coccinellidae), is a common predatory coccinellid found in a wide range of natural and agricultural habitats of the world including China, Japan, Korea and Pakistan, and prefers to feed on insect pests that belonging to Aphidoidea, Psylloidea, Coccoidea, and mites. It is a successful predator both in arid and semi-arid regions because of its tolerance to high temperature and capability to reproduce under nutritionally poor quality prey and pollen supplements. P. japonica also remains active throughout the year, without undergoing aestivation, and completes 3-4 and 7-8 generations in Northern and Southern China, respectively. Both stages of the beetle, larvae and adults are predacious with a very high rate of daily prey consumption and, thus, play a considerable role in suppression of economic pests (Luo et al. 2014). Many studies demonstrated that the $B t$ proteins do not pose any adverse effect on the NTAs. However, few studies reflect deleterious effects of $B t$ proteins on some NTAs (Liu et al. 2016).

Non-target arthropods such as $P$. japonica are usually exposed to plant expressed $B t$ proteins when feeding on prey and pollens in GM crops. Therefore, it is needed that bio-safety of $B t$ protein such as Vip3Aa must be evaluated to ensure safety of GM crops expressing Vip3Aa. $P$. japonica being a sensitive and easy to be reared in the laboratory is considered as the model test insect for studying the potential adverse impacts of GM crops and pesticides before their registration ( $\mathrm{Li}$ et al. 2017). It is also being mass reared on natural and factitious prey under controlled laboratory conditions to be used in inoculative and augmentative biological control programs (Mirkhalilzadeh et al. 2013).

Zhang et al. (2013) reported 400 ng concentration of Cry2Aa in P. japonica collected from the GM rice and also assessed the ecological effects of two
Bt cotton cultivars, ZMS and ZMKCKC, expressing Cry1Ac/Cry2Ab and Cry1Ac/EPSPS, respectively, on P. japonica through its herbivorous prey, Aphis gossypii (Glov.) and found that GM cotton cultivars had no detrimental effects on the biology of P. japonica. In the present study, potential adverse effects of Vip3Aa protein which transgene exhibited into some GM crops were being evaluated on the larval development, mortality and adult weight of $P$. japonica.

\section{Methods \\ Insects}

Propylea japonica adults were collected from the research farm of Cotton Research Institute, Chinese Academy of Agricultural Sciences (CRI-CAAS) Anyang, $\left(36^{\circ} \mathrm{N}, 114^{\circ} \mathrm{E}, \mathrm{HSL}: 69 \mathrm{~m}\right)$ Henan, China, and were reared on the pea aphid (Acyrthosiphon pisum [Harris]) in acrylic rearing chamber $(45 \times 45 \times 25 \mathrm{~cm})$ in the laboratory under controlled conditions. The pea aphid was reared on potted broad bean, Vicia faba seedlings. Young larvae ( $12 \mathrm{~h}$-old) of $P$. japonica were used in the experiment.

\section{Bio-insecticidal compounds}

The bio-insecticidal compounds used in this study were as follows: Bt protein, Vip3Aa (95-98\% pure) and boric acid (99.5\%). Bt protein Vip3Aa was purchased from Envirotest-China (an agent for EnviroLogix, Portland, ME). A Vip3A clone in Escherichia coli was used as a source of Vip3Aa toxin production. The methods used to express and purified the Vip3Aa toxin generally, followed procedure described by Chen et al. (2002). The purity of Vip3Aa was estimated, using SDS-polyacrylamide gel electrophoresis. Activated protein was stored at $-80{ }^{\circ} \mathrm{C}$ in the laboratory. Boric acid was purchased from Sigma-Aldrich. The median effective concentration $\left(\mathrm{EC}_{50}\right)$ of Vip3Aa was determined about $500 \mathrm{ng} / \mathrm{g}$, using bioassay previously described by Ali et al. (2017). Briefly, newly emerged first instar larvae of a susceptible strain of Helicoverpa armigera (Hübner) (Lepidoptera: Noctuidae) were exposed to various concentrations of Vip3Aa protein ranging from 100 to $500 \mathrm{ng} / \mathrm{g}$ synthetic diet for 7 days and $\mathrm{EC}_{50}$ (Effective pathogenic concentration that results in $50 \%$ weight reduction in the tested insect compared to that of the control) was estimated comparing larval weights to control treatment.

\section{Synthetic diet-based rearing and dietary exposure test}

The semi-solid synthetic diet previously used to rear the larvae of Harmonia axyridis (Pallas) and P. japonica was used to rear and expose P. japonica to elevated concentration of $B t$ protein (Ali et al. 2016). Diet was 
freshly prepared using beef, beef liver, egg yolk, yeast extract, honey, sucrose, royal jelly, ascorbic acid, vitamins powder and preservative in distilled water, and ingredients were thoroughly mixed in a blender to achieve semi-solid consistency. Bt protein Vip3Aa was added. Boric acid added diet served as positive control while pure synthetic diet served as negative control. Diet was stored at $-20^{\circ} \mathrm{C}$.

In order to expose $P$. japonica to $B t$ protein, young larvae ( ${ }^{<} 12 \mathrm{~h}$-old) were individually reared in 8 -ml centrifuge tubes having 2 holes for ventilation and fed on synthetic diet containing Vip3Aa, boric acid as positive control and pure synthetic diet as the negative control. The nominal concentration of Vip3Aa protein incorporated in the diet was $200 \mu \mathrm{g} / \mathrm{g}$ of fresh diet whereas the boric acid: a stomach poison was used as a positive control to indicate the validity of experiment to test effects of toxin incorporated into diet. The nominal concentration of boric acid incorporated into the fresh synthetic diet was $50 \mu \mathrm{g} / \mathrm{g}$ (Ali et al. 2017). Every day fresh diet was provided twice as small droplets to the immature $P$. japonica with the help of a syringe during larval development until larvae pupated or died in all treatment.

Seventy young larvae of $P$. japonica were reared in each treatment and biological parameters like survival, pupation, larval development and 7-day larval weights were recorded. Emerged adults were weighed (within $12 \mathrm{~h}$ ), sexed, and then stored at $-80^{\circ} \mathrm{C}$ to perform ELISA test. The adult weights were compared separately based on their sex. The bioassay was considered terminated when the larvae in all treatments either developed into adults or died.

\section{Stability of Vip3Aa in synthetic diet}

Stability of Vip3Aa was estimated in five samples (20$30 \mathrm{mg}$ ) drawn from the diet before and after exposure to the ladybird beetle, using double-antibody sandwich enzyme-linked immunosorbent assays (DAS-ELISA) as previously described by Ali et al. (2017). Briefly, all insects were washed in Phosphate-buffered saline Tween (PBST) to remove Vip3Aa toxin from their body surface. For Vip3Aa protein extraction, insects were weighted and mixed with PBST at a ratio 1:100 (mg sample: $\mu \mathrm{l}$ buffer) in 5-ml centrifuge tubes. Subsequently, samples were fully ground using glass grinding tube and pestle. After centrifugation at $4.66 \times 10^{3} \mathrm{~g}$ for $10 \mathrm{~min}$ at $4{ }^{\circ} \mathrm{C}$, the supernatants obtained were diluted to measureable concentration and ELISA (EnviroLogix Portland, Maine, USA) was performed according to the manufacturer's instructions. The optical density was measured at wavelength $450 \mathrm{~nm}$ and calibrated to a range of standards made from purified toxin to estimate $B t$ toxin.

\section{Detection of Vip3Aa in the emerged adults}

Uptake of Vip3Aa was determined in 5 insects randomly selected from the insects reared in each treatment by using ELISA technique as described above.

\section{Bioactivity verification of Vip3Aa}

Larvae of $B t$-susceptible strain of $H$. armigera already maintained in the laboratory were used as test insect to verify the bioactivity of Vip3Aa. The supernatant used for the ELISA was diluted to the extent of the estimated $\mathrm{EC}_{50}$ (500 ng/g fresh diet) and then incorporated into the synthetic diet used to rear the larvae of $H$. armigera (Zhou et al. 1981). Newly emerged first instar larvae of $H$. armigera were individually kept into multi-well culture plates covered with a thick cloth and lid to prevent escape. Sixty $H$. armigera larvae were fed on synthetic diet containing supernatant for 7 days and larvae were weighed to assess bioactivity in terms of reduced larval weight compared to the control treatment (pure synthetic diet).

\section{Data analyses}

Pupation and adult emergence rates of $P$. japonica were analyzed using chi-square test, while larval development times, the male and female adult weights were analyzed using Mann-Whitney $U$ test as data followed non-normal distribution. Larval weights of $H$. armigera reared on pure synthetic diet and synthetic diet containing supernatant were compared using student's $t$ test. Similarly, concentrations of Vip3Aa in the fresh diet and diet exposed for $12 \mathrm{~h}$ feeding were analyzed using student's $t$ test. All the statistical analyses were performed using SPSS ver. 20 software package for the Windows 2007 (SPSS, Inc., Chicago, IL, USA).

\section{Results}

Impact of Vip3Aa on the life table parameters of P. japonica The results indicated that about $57-60 \%$ larvae successfully completed their development and successfully pupated in the negative control and Vip3Aa treatments, which did not differ significantly (Fig. 1). Similarly emergence rate was above $85 \%$ among the pupated larvae in both treatments. Likewise, the male and female fresh weights did not vary significantly from that of the negative control (Table 1). However, the time taken by the larvae to complete larval development was significantly prolonged in the Vip3Aa treatment than that of the negative control (Table 1). In contrast, positive control when larvae were nourished on synthetic diet containing boric acid all the larvae died during their earlier developmental instars. Therefore, this treatment was excluded from analysis (Table 1). Death of larvae in their earlier 


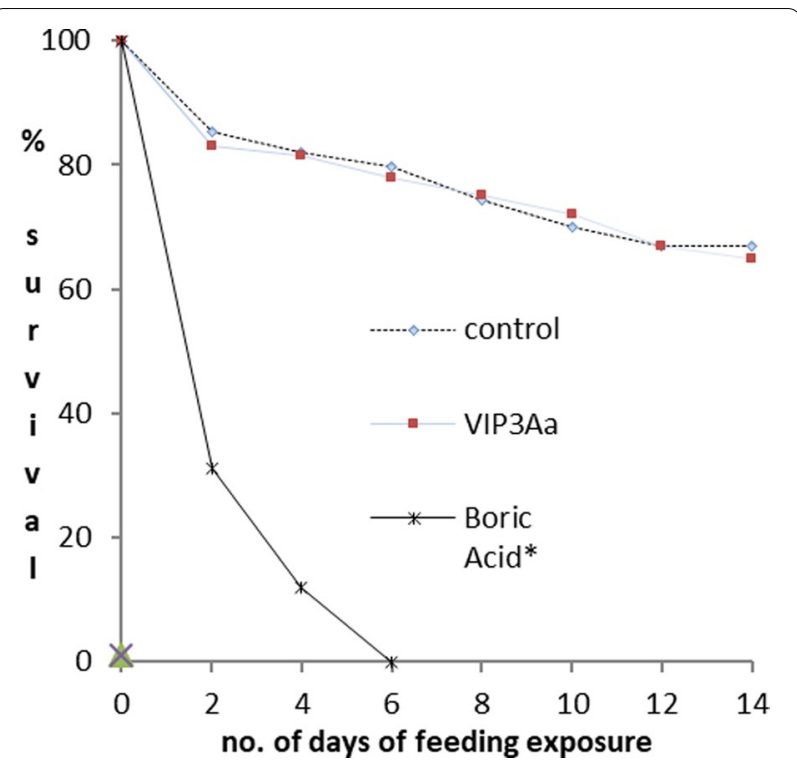

Fig.1 Impact of Vip3Aa on the survival of immature Propylea japonica

developmental instar in positive control indicated the sensitivity of the bioassay to detect any adverse effect of insecticide incorporated in diet on the test organism.

\section{Vip3Aa stability}

The reliability of test's results also depends on whether the protein in question is biologically active and remains stable during diet preparation and diet storage. The ingestion factor also plays a crucial role in deciding the biosafety of protein to non-target arthropods. The results of the present study showed that Vip3Aa remained stable when incorporated into the synthetic diet during diet preparation and storage. The mean concentrations of Vip3Aa protein detected in the fresh diet and in diet after $12 \mathrm{~h}$. of feeding exposure were 136.83 and $113.14 \mu \mathrm{g} / \mathrm{g}$, respectively. This difference in the degradation rate (17\%) was non-significant (student's $t$ test; $t=1.06 ; d f=6$; $p=0.33$ ) (Fig. 2a). Consequently, the protein remained stable during the diet preparation and feeding exposure to avoid false negative results.

\section{Bioactivity verification and detection of Vip3Aa in emerged adults}

Bioactivity of the Vip3Aa was confirmed by exposing the young larvae of susceptible $H$. armigera to the protein. The results indicated that when susceptible newly emerged first instar larvae of $H$. armigera were fed for 7 days on the diets containing supernatants extracted from fresh and exposed synthetic diets containing Vip3Aa protein, the mean fresh larval weight $(1.41 \mathrm{~g})$ of $H$. armigera was reduced significantly than the control (4.87 g) (student's $t$ test; $t=6.59, d f=91, p=0.00$ ) (Fig. 2b). In the present study, average concentration of ingested Vip3Aa protein in the emerged adults of $P$. japonica was also measured, using DAS-ELISA which was $178.56 \mathrm{ng} / \mathrm{g}$ of fresh body weight, whereas no Vip3Aa was detected in the emerged adults in the control treatment.

\section{Discussion}

Genetically modified crops producing $B t$ endotoxins are planted worldwide to control the target insect pests particularly those belonging to the order Lepidoptera (e.g., Helicoverpa zea, $H$. armigera). However, their prolific increase in plantation expose the NTAs including pollinators, predators and parasitoids to the pathogenic concentration that may prove lethal at the highest trophic level and may reduce their population as well as their services as pollinator and biological control agents (Harwood et al. 2005). Therefore, it is considered obligatory to test the effects of GM crops on NTAs before issuing approval for their commercial plantation in the field.

Table 1 Impact of Vip3Aa and boric acid on the biology of immature Propylea japonica

\begin{tabular}{|c|c|c|c|c|c|}
\hline \multirow[t]{2}{*}{ Treatment } & \multirow[t]{2}{*}{ Pupation (\%) } & \multirow[t]{2}{*}{ Emergence (\%) ${ }^{\mathrm{a}}$} & \multirow[t]{2}{*}{ Larval duration $(\mathbf{d} \pm \mathrm{SE})^{\mathbf{b}}$} & \multicolumn{2}{|c|}{ Adult fresh weight $(\mathrm{mg} \pm \mathrm{SE})^{\mathrm{b}}$} \\
\hline & & & & Male & Female \\
\hline Control (Negative) & $60(42)^{\mathrm{a}}$ & $100(42)^{\mathrm{a}}$ & $10.21 \pm 1.14(52)^{\mathrm{a}}$ & $2.66 \pm 0.09(19)^{\mathrm{a}}$ & $3.75 \pm 0.69(23)^{a}$ \\
\hline Vip3Aa & $57(40)^{a}$ & $85(34)^{\mathrm{a}}$ & $12.70 \pm 0.88(40)^{b}$ & $2.87 \pm 0.12(17)^{a}$ & $3.86 \pm 0.32(17)^{\mathrm{a}}$ \\
\hline Boric acid (Positive) & - & - & - & - & - \\
\hline Control vs Vip3Aa & $\begin{array}{l}x^{2}=2.00 \\
d f=2 \\
p=0.1368\end{array}$ & $\begin{array}{l}x^{2}=4.00 \\
d f=2 \\
p=0.135\end{array}$ & $\begin{array}{l}Z=-7.81 \\
P=0.000\end{array}$ & $\begin{array}{l}Z=-1.73 \\
P=0.087\end{array}$ & $\begin{array}{l}Z=-1.51 \\
P=0.130\end{array}$ \\
\hline
\end{tabular}

Same letters in the column indicate the mean are significantly similar while with different letters indicate significant difference among treatments. However all larvae died in positive control indicated in table as -. Therefore this treatment is excluded from the analysis

${ }^{\text {a }}$ Chi-square test

${ }^{\mathrm{b}}$ Mann-Whitney $U$ test 

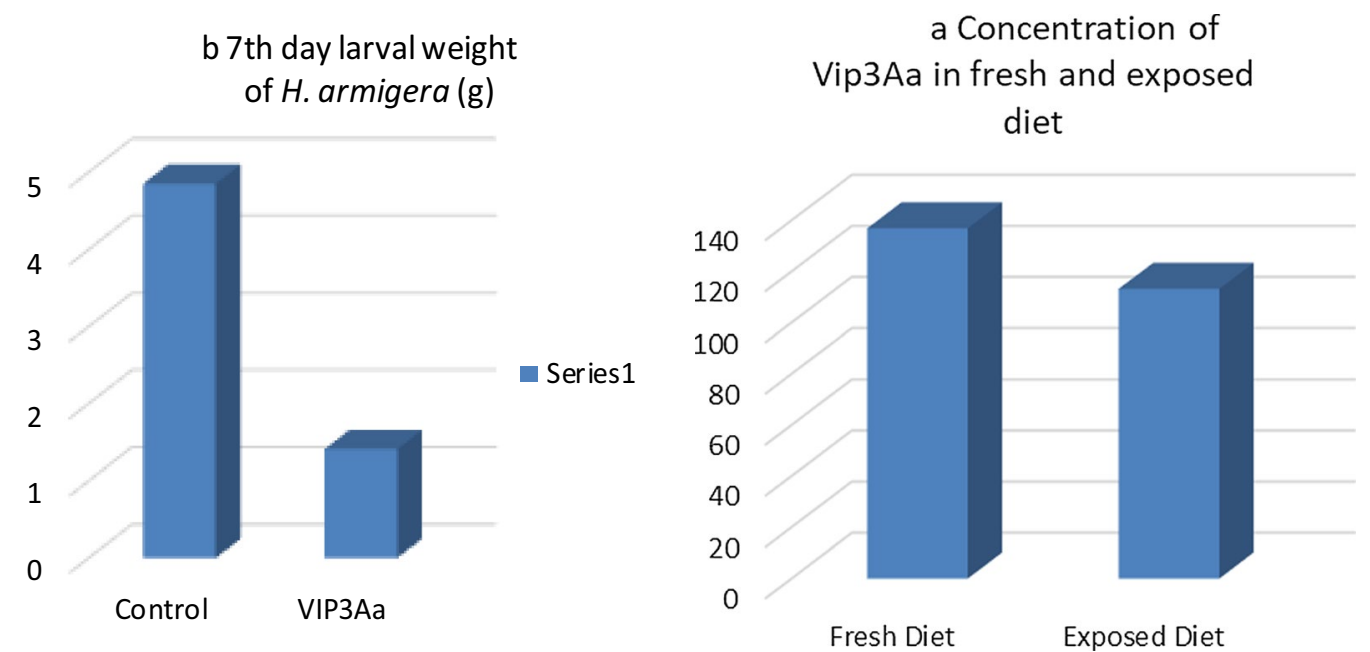

Fig. 2 a Concentration of Vip3Aa $(\mu \mathrm{g} / \mathrm{g})$ detected in fresh and exposed diets. $\mathbf{b}$ 7-day post-treatments larval weight of Helicoverpa armigera larvae fed diet with and without Vip3Aa

For this purpose, a project was designed to evaluate the potential adverse effects of various $B t$ proteins on NTAs and this experiment was the part of that project which emphasized to assess the direct effects of Vip3Aa protein on life table parameters of $P$. japonica an abundant ladybird in China and neighboring countries. Many previous studies highlighted no adverse effects of $B t$ proteins on the NTA, However, few studies also exhibit mild adverse effects of $B t$ proteins on the NTA. These studies indicated mild side effects, when the NTAs were fed on the preys reared on the $B t$ crops. For instance, developmental delays were observed when Chrysoperla carnea (Steph.) were fed on the Spodoptera littoralis (Boisd.) larvae reared on the $B t$ maize expressing Cry1Ab (Dutton et al. 2002). Similarly, developmental delays were observed when Chrysoperla sinica (Tjeder) were fed on Bemisia tabaci (Genn.) reared on Bt cotton producing Cry1Ac (Guo et al. 2004). However, there are also some studies that differed from these findings and hinted that $B t$ proteins had none of the apparent adverse effects on the survival of green lace wing larvae (Wang et al. 2012) or other NTAs when administered through direct incorporation into the synthetic diet or indirectly through prey (Tian et al. 2018). Shera et al. (2018) studied the effects of single toxin (Cry 1Ac) and dual toxin (CrylAc and Cry 2Ab) on the fitness parameters of Chrysoperla zastrowi, sillemi (Esben-Peterson), a generalist predator in the cotton ecosystem, through its herbivore preys such as mealy bug, whitefly and leaf hoppers and concluded that both proteins were proved apparently safe on test species. Likewise, in another test case, biological parameters such as larval development, survival and larval weight did not differ in the ladybird beetle Stethorus punctillum (Weise) fed on the spider mites reared on $B t$ and non- $B t$ maize (Li and Romeis 2010). Tian et al. (2018) examined the effects of $B t$ cotton containing Cry1 Ac and Cry2Ab on the Cotesia marginiventris (Cresson) an important parasitoid of noctuid pests, eliminating the host quality effects and concluded that $B t$ cotton had no detrimental effect on the development, mummy weight and progeny produced. The results of present experiment are similar to the above studies; however, mild delay was noted in larval developmental time, while all other life parameters remained unchanged indicating no prominent side effect of Vip3Aa on P. japonica.

The results of the experiment seem to be over-estimated rather than under-estimated because quantity of protein administered via diet $(200 \mu \mathrm{g} / \mathrm{g}$ of fresh diet) to Japanese beetle was 10 times higher than that quantity which may be possibly ingested by the beetle consuming pollens and preys in the GM crops. For instance, Vip3Aa concentration measured in MIR 162 (Corn) varied from $17.35-24.62 \mu \mathrm{g} / \mathrm{g}$ to $50.41-56.56 \mu \mathrm{g} / \mathrm{g}$ in the whole plant and leaves, respectively (Raybould and Vlachos 2011). Ladybird beetle may possibly consume $20 \%$ of the highest expressed concentration in plants while consuming pollen and prey.

\section{Conclusions}

It is concluded that immature $P$. japonica was found insensitive to Vip3Aa and mild adverse effects observed during development at much higher concentration are not possible in field conditions where protein ingestion level is potentially very low, while consuming pollen and preys in a Bt-crop. It is further concluded on the bases of results of the present experiment that $B t$ crops expressing 
Vip3Aa are not harmful to the non-target organisms and can be integrated in the biological pest control programs as an integrated pest management strategy.

\author{
Abbreviations \\ Bt: Bacillus thuringiensis; Cry: Crystalline; d: Day; DAS-ELISA: Double-antibody \\ sandwich enzyme-linked immunosorbent assay; $\mathrm{EC}_{50}$ : Effective concentrate \\ 50; HSL: Height from sea level; GM: Genetically modified; IL: Illinois; Inc: Incor- \\ poration; NTA: Non-target arthropods; PBST: Phosphate buffer saline tween; \\ SE: Standard error; SPSS: Statistical package for social sciences; VIP: Vegetative \\ insecticidal protein.
}

\section{Acknowledgements}

We say special thanks to anonymous reviewers for their valuable comments on the first draft of the manuscript.

\section{Authors' contributions}

IA, conceived of, designed and performed the experiments. He also wrote the paper and analyzed the data. SZ and JC contributed to the reagents/materials/analysis tools. Other authors help in refining the manuscript. All authors read and approved the final manuscript.

\section{Funding}

This study was funded by the Transgenic Major Projects Program of the Ministry of Science and Technology, China (2014ZX08011-002) and the Chinese Scholarship Council.

\section{Availability of data and materials}

All data generated or analyzed during this study are included in this article.

\section{Declarations}

Ethics approval and consent to participate

Not applicable.

\section{Consent for publication}

Not applicable.

\section{Competing interests}

The authors declare that they have no competing interests.

\section{Author details}

${ }^{1}$ State Key Laboratory of Cotton Biology, Institute of Cotton Research, Chinese Academy of Agricultural Sciences, Anyang 455000, China. ${ }^{2}$ Department of Entomology, Faculty of Agriculture and Environment, The Islamia University of Bahawalpur, Baghdad UI Jadeed Campus, Bahawalpur 63100, Pakistan. ${ }^{3}$ Faculty of Agriculture, Lasbela University of Agriculture, Water and Marine Sciences, (LUAWMS), Uthal 90150, Baluchistan, Pakistan. ${ }^{4}$ College of Horticulture and Plant Protection, Yangzhou University, Yangzhou, China. ${ }^{5}$ Department of Plant Protection, Ministry of National Food Security and Research, Islamabad, Pakistan. ${ }^{6}$ Department of Plant Protection, College of Food and Agriculture Sciences, King Saud University, Riyadh 11451, Saudi Arabia.

\section{Received: 28 April 2021 Accepted: 6 November 2021}

Published online: 23 November 2021

\section{References}

Ali I, Zhang S, Luo J-Y, Wang C-Y, Lv L-M, Cui J-J (2016) Artificial diet development and its effect on the reproductive performances of Propylea japonica and Harmonia axyridis. J Asia-Pac Entomol 19:289-293

Ali I, Zhang S, Cui JJ (2017) Bio-safety evaluation of Cry1Ac, Cry2Ab, Cry1Ca, Cry1F and Vip3Aa on Harmonia axyridis larvae. J Appl Entomol 141:53-60

Chakrabarty S, Jin M, Wu C, Chakraborty P, Xiao Y (2020) Bacillus thuringiensis vegetative insecticidal protein family Vip3A and mode of action against pest Lepidoptera. Pest Manag Sci 76:1612-1617
Chen J-W, Tang L-X, Tang M-J, Shi Y-X, Pang Y (2002) Cloning and expression product of vip3A gene from Bacillus thuringiensis and analysis of inseceicidal activity. Chin J Biotechnol 18:687-692

Dutton A, Klein H, Romeis J, Bigler F (2002) Uptake of Bt-toxin by herbivores feeding on transgenic maize and consequences for the predator Chrysoperla carnea. Ecol Entomol 27:441-447

Guo J-Y, Wan F-H, Dong L (2004) Survival and development of immature Chrysopa sinica and Propylciea japonica feeding on Bemisia tabaci propagated on transgenic Bt cotton. Chin J Biol Control 20:164-169

Harwood JD, Wallin WG, Obrycki JJ (2005) Uptake of Bt endotoxins by nontarget herbivores and higher order arthropod predators: molecular evidence from a transgenic corn agroecosystem. Mol Ecol 14:2815-2823

Jafar W, Mazlan N, Adam NA, Omar D (2013) Evaluation on the effects of insecticides on biodiversity of arthropod in rice ecosystem. Acta Biol Malays 2:115-123

Klümper W, Qaim M (2014) A meta-analysis of the impacts of genetically modified crops. PLOS ONE 9:e111629

Kumar K, Gambhir G, Dass A, Tripathi AK, Singh A, Jha AK, Yadava P, Choudhary M, Rakshit S (2020) Genetically modified crops: current status and future prospects. Planta 251:1-27

Li Y, Romeis J (2010) Bt maize expressing Cry3Bb1 does not harm the spider mite, Tetranychus urticae, or its ladybird beetle predator, Stethorus punctillum. Biol Control 53:337-344

Li Y, Zhang Q, Liu Q, Meissle M, Yang Y, Wang Y, Hua H, Chen X, Peng Y, Romeis $J$ (2017) Bt rice in China-focusing the nontarget risk assessment. Plant Biotechnol J 15:1340-1345

Liu Y, Liu Q, Wang Y, Chen X, Song X, Romeis J, Li Y, Peng Y (2016) Ingestion of Bt corn pollen containing Cry1 Ab/2Aj or Cry1Ac does not harm Propylea japonica larvae. Sci Rep 6:1-7

Luo S, Naranjo SE, Wu K (2014) Biological control of cotton pests in China. Biol Control 68:6-14

Mirkhalilzadeh RE, Allahyari H, Nozari J, Farhoudi F (2013) Rearing larval stages of Hippodamia variegata Goeze (Coleoptera: Coccinellidae) on artificial diet. Arch Phytopathol Plant Prot 46:755-765

Palma L, Muñoz D, Berry C, Murillo J, Caballero P (2014) Bacillus thuringiensis toxins: an overview of their biocidal activity. Toxins 6:3296-3325

Raybould A, Vlachos D (2011) Non-target organism effects tests on Vip3A and their application to the ecological risk assessment for cultivation of MIR162 maize. Transgenic Res 20:599-611

Romeis J, Bartsch D, Bigler F, Candolfi MP, Gielkens MM, Hartley SE, Hellmich RL, Huesing JE, Jepson PC, Layton R, Quemada H, Raybould A, Rose RI, Schiemann J, Sears MK, Shelton AM, Sweet J, Vaituzis Z, Wolt JD (2008) Assessment of risk of insect-resistant transgenic crops to nontarget arthropods. Nat Biotechnol 26:203-208. https://doi.org/10.1038/nbt1381

Shera PS, Karmakar P, Sharma S, Sangha KS (2018) Impact of Bt cotton expressing single ( $\mathrm{Cry} 1 \mathrm{Ac}$ ) and dual toxins (Cry1 $\mathrm{Ac}$ and $\mathrm{Cry} 2 \mathrm{Ab})$ on the fitness of the predator Chrysoperla zastrowi sillemi (Esben-Petersen): prey-mediated tri-trophic analysis. Egypt J Bio Pest Control 28:98

Tian J-C, Wang X-P, Chen Y, Romeis J, Naranjo SE, Hellmich RL, Wang P, Shelton AM (2018) Bt cotton producing CrylAC and Cry2Ab does not harm two parasitoids, Cotesia marginiventris and Copidosoma floridanum. Sci Rep 8:307

Wang Y, Li Y, Romeis J, Chen X, Zhang J, Chen H, Peng Y (2012) Consumption of Bt rice pollen expressing Cry2Aa does not cause adverse effects on adult Chrysoperla sinica Tjeder (Neuroptera: Chrysopidae). Biol Control 61:246-251

Yin Y, Xu Y, Cao K, Qin Z, Zhao X, Dong X, Shi W (2020) Impact assessment of $\mathrm{Bt}$ maize expressing the Cry1 $\mathrm{Ab}$ and Cry2Ab protein simultaneously on non-target arthropods. Environ Sci Pollut Res 27:21552-21559

Zhang S-Z, Zhang F, Hua B-Z (2007) Suitability of various prey types for the development of Propylea japonica (Coleoptera: Coccinellidae). Euro J Entomol 104:149

Zhang Q, Li Y, Hua H, Yang C, Wu H, Peng Y (2013) Exposure degree of important non-target arthropods to CryzAa in Bt rice fields. J Appl Ecol 24:1647-1651

Zhou L, Fang Y, Yang J (1981) Investigation on artificial diet in Heliothis armigera. Acta Entomol Sin 24:108-110

\section{Publisher's Note}

Springer Nature remains neutral with regard to jurisdictional claims in published maps and institutional affiliations. 